\title{
DNA Repair Protein OGG1 in Pulmonary Infection and Other Inflammatory Lung Diseases
}

\author{
Ping Lin, Qinqin Pu, Shugang Qin, Jacob Schettler, \\ Mariah Thoemke, Guoping Li, Jianxin Jiang, and Min Wu
}

\begin{abstract}
In the last decades, extensive research has uncovered functional roles and underlying mechanisms of DNA repair enzyme 8-oxoguanine DNA glycosylase (OGG1) in the pathogenesis of inflammatory response in infection and other diseases in the lung. OGG1 excises 8-oxo-7,8-dihydroguanine (8-oxo-dG) lesion on DNA that is often induced by generation of reactive oxygen species (ROS) and has been linked to mutations, cancer development, and tissue damage. Most, if not all, environmental toxic agents and mammalian cellular metabolites elicit the generation of ROS, either directly, indirectly, or both, which is among the first cellular responses. ROS in combination with other oxidative molecules/moieties are recognized as a major factor for killing invading pathogens but meanwhile can cause tissue damage. ROS potentially modify proteins, lipids, and
\end{abstract}

Authors Ping Lin, Qinqin Pu and Shugang Qin have been equally contributed in this work.

\section{P. Lin}

Department of Biomedical Sciences, School of Medicine and Health Sciences,

University of North Dakota, Grand Forks, ND, USA

State Key Laboratory of Trauma, Burns and Combined Injury, Institute of Surgery Research, Daping Hospital, Army Medical University, Chongqing, People's Republic of China

Q. Pu · S. Qin · J. Schettler · M. Thoemke · M. Wu (ه)

Department of Biomedical Sciences, School of Medicine and Health Sciences,

University of North Dakota, Grand Forks, ND, USA

e-mail:min.wu@med.und.edu

G. Li

Pulmonary and Allergy Institute, Affiliated Hospital, Southwestern Medical University,

Luzhou, China

\section{J. Jiang}

State Key Laboratory of Trauma, Burns and Combined Injury, Institute of Surgery Research, Daping Hospital, Army Medical University, Chongqing, People's Republic of China 
DNA due to the strong molecular reactivity. While oxidative stress causes increased levels of all types of oxidatively modified DNA bases, accumulation of 8-oxo-dG in the DNA has been singled out to be a main culprit linking to various inflammatory disease processes. Oxidatively damaged DNA bases such as 8-oxo$\mathrm{dG}$ are primarily repaired by the base excision repair (BER) mechanism, in which OGG1, as the lesion recognition enzyme, plays a fundamental role in fixing this DNA damage. In this chapter, we summarize the roles and potential mechanistic analyses of OGG1 in lung infection and other inflammatory diseases.

\subsection{Introduction}

The lung is the vital organ in the body to supply oxygen and remove carbon dioxide and is vulnerable to exogenous sources of oxidative stress because of the continuous, direct exposure to the external environment (Vlahopoulos et al. 2018). Pulmonary infection often has a consequence of inflammatory response in the lung parenchyma including the terminal airway, alveolar cavity, and interstitial lung. A variety of pathogens including bacteria, viruses, and fungi are the most common causal agents in lung diseases (Stehle et al. 2018). Bacterial infection can lead to serious lung injury by initiating oxidative damage and inflammatory responses to activate a series of signaling pathways (Liu et al. 2018). Reactive oxygen species (ROS) contain a spectrum of oxidative products that aerobic cells produce during metabolism, including $\mathrm{O}_{2}^{-}, \mathrm{H}_{2} \mathrm{O}_{2}, \mathrm{HO}_{2}{ }^{-},-\mathrm{OH}$, and so on (Ewald 2018). A great deal of research has uncovered that low concentrations of ROS can beneficially regulate a number of signal transduction pathways in cells, while medium and high concentrations of ROS induce apoptosis and necrosis through cellular oxidative stress. The two-way regulation of ROS is essential for maintaining the metabolic balance of cells (Testa et al. 2018). Imbalance of ROS production will lead to tumorigenesis and disease progression (Yuan et al. 2017). The occurrence of lung disease is closely related to levels of ROS in vivo when exposed to environmental stimuli, such as bacteria, chemicals, oxygens, etc. ROS accumulation in cells induces DNA oxidative damage associated with lung diseases including pneumonia (Zhang et al. 2018). Among the several ROS-induced DNA base lesions in the genome, 8-oxo-7,8dihydroguanine (8-oxo-dG) is one of the most abundant because guanine is extremely susceptible to oxidative attack. DNA repair enzyme 8-oxoguanine DNA glycosylase (OGG1) specifically repairs damaged DNA which arises when exposed to ROS to prevent 8-oxo-dG accumulation and maintain cell genetic stability. 8-Oxo-dG has been linked to the pathogenesis of bacterial infection and other inflammatory lung diseases (Ba et al. 2014). OGG1 repairs damaged DNA mainly by initiating DNA base excision repair (BER) pathway. First, OGG1, a DNA glycosylase/AP lyase, hydrolyzes $\mathrm{N}$-glycosyl sites to release 8-oxo as a free base, which then cleaves the sugar phosphate backbone to form a depurination/depyrimidine (AP) site. OGG1 would then recruit depurination/apyrimidine endonuclease 1 
(APE1) to remove the $3^{\prime}$-phosphate- $\alpha, \beta$-unsaturated group and form a 3'-OH end. DNA polymerase $\beta$ carries the correct guanine insertion gap and recruits ligase II/ $\mathrm{XRCC} 1$ linkage. The cleaved 8 -oxo-dG base is released into the cytoplasm and is bound by OGG1 to form OGG1-8-oxoG complex to activate GDP $\rightarrow$ GTP. These actions may require a series of regulators involving multiple DNA repair enzymes and signaling proteins within the cell (Wang et al. 2018). The expression of OGG1 is an important marker of lung infection and inflammatory processes resulted from accumulation of ROS levels or other potential stress factors (Zhang et al. 2017).

The dual role of DNA repair enzyme OGG1 in innate immunity and lung diseases allows rapid fixation of base damage caused by different types of bacteria and external stimuli. Our recent research showed that $\operatorname{Ogg~}^{-/-}$mice exhibit significantly challenged inflammatory response including cell infiltration after challenge of ovalbumin (OVA), a reagent that causes asthmatic pathology ( $\mathrm{Li}$ et al. 2012). However, $O g g 1^{-/-}$mice exhibited increased lung injury after infection with P. aeruginosa ${ }^{12}$. OGG1 is of importance in repairing oxidative DNA damage and activating a range of signaling pathways. In this section, we describe the roles and related mechanisms of what we call the DNA Repair Protein OGG1 Response (DRPOR) to lung infection and inflammatory diseases.

\subsection{DNA Repair Protein OGG1 Response to Lung Infection}

\subsubsection{OGG1 Plays a Role in Bacterial Infection}

\subsubsection{Pseudomonas aeruginosa}

A certain concentration of ROS induces apoptosis or necrosis through different signal transduction pathways, such as oxidative stress response. Oxidative stress responses lead to DNA fragmentation, DNA site mutation, and double-strand distortion, while DNA repair mechanism plays critical roles in host defense against oxidative stress resulting from various ruthless attacks. The opportunistic pathogen P. aeruginosa causes host ROS release during infection by releasing quorum sensing (QS) molecules and inducing pronounced DNA damage. As mitochondrial DNA (mtDNA) is highly sensitive to ROS/RNS (reactive nitrogen species), oxidative mtDNA was found to damage the lung and cause $P$. aeruginosa-induced acute lung injury(Lee et al. 2017). The $P$. aeruginosa caused oxidative mtDNA damage and then resulted in a feedback cycle of mtDNA damage and DNA damageassociated molecular pattern (DAMP) formation that leads to consequences of acute lung injury(Kuck et al. 2015). As OGG1 is the indispensable DNA repair enzyme and its expression and enzymatic activity was found to be strikingly changed in lungs, Wu et al. demonstrated that the OGG1 acetylation site mutation exacerbated Cockayne syndrome group B (CSB) expression and found that deletion of OGG1 results in lung injury during $P$. aeruginosa infection. Coincidentally, Cheng and colleagues confirmed that deacetylase sirtuin 3 (Sirt3) could slow down the degradation of OGG1 to protect mtDNA damage under oxidative stress(Wu et al. 2011; 
Cheng et al. 2013). Thus, OGG1 likely plays a key role in response to $P$. aeruginosa infection via acetylation-associated signaling.

\subsubsection{Klebsiella pneumoniae}

$K$. pneumoniae is one of the human iatrogenic bacteria and one of the fundamental causes of pulmonary infections and sepsis. Huang et al. found that OGG1 overexerted lung cells to alleviate cytotoxicity during $K$. pneumoniae infection, suggesting that OGG1 affects DNA damage and repair through membrane lipid-mediated signal axis against $K$. pneumoniae. Mechanistically, infection-induced oxidation causes DNA damage following ROS induction in lung cells through activation of lipid rafts, which is from a totally new angle to explain how OGG1 fixes DNA damage through lipid raft-mediated pathways(Huang et al. 2013). Although more studies are required to further understand the role of OGG1 in coordinating a concerted regulation of immune response in $K$. pneumoniae lung infection, understanding of lipid raft-associated pathways may provide better views of OGG1's involvement in host response to K. pneumoniae infection.

\subsubsection{Other Bacteria}

Staphylococcus aureus pneumonia is an acute pulmonary purulent inflammation caused by $S$. aureus. S. aureus is highly toxic by producing hemolytic toxins, plasma coagulase, and deoxyribonucleic acid catabolism enzyme and has a serious threat to children's lives. Bartz et al. (Bartz et al. 2011) are a major team in studying OGG1 and mtDNA repair during $S$. aureus infection. Although their studies are directed to sepsis and tested tissue injury in the kidney and liver rather than the lung, the mechanism they found may reflect other organs including the lungs. In their studies, mtDNA repair and mitochondrial biogenesis were evidenced by increased levels of OGG1, nuclear respiratory factor (NRF), and mitochondrial transcription factor A expression (Bartz et al. 2011, 2014). NRF is well known to play key roles in regulating mitochondrial biogenesis, which is vital for ROS production during lung infection. Hence, the function of OGG1 may be important for host defense to control $S$. aureus-induced oxidative damage.

Helicobacter pylori is also a gram-negative bacterium like $P$. aeruginosa, mainly distributed in gastric mucosa tissues, and 67-80\% of gastric ulcers and 95\% duodenal ulcers are caused by H. pylori (Kate et al. 2013). Earlier, H. pylori was thought to just exist in the stomach, but now studies have suggested that $H$. pylori also exists in the mucosa of the respiratory tract(Deng et al. 2013), which may be involved in some lung diseases including pulmonary tuberculosis and chronic bronchitis(Adriani et al. 2014). Although there is no study reported in OGG1 to H. pylori in the lung, OGG1 is constantly linked to repairing DNA under $H$. pylori infection in other organs (Touati et al. 2006; Mathieu et al. 2006) (Izzotti et al. 2007). It is possible that OGG1 plays a critical role in lung disease associated with $H$. pylori too. Finally, it will be interesting to understand the involvement and mechanisms of OGG1's function during infection by Escherichia coli, Streptococcus pneumoniae, Haemophilus influenzae, and Legionnaires' disease, which are associated with lung infections (Fig. 4.1). 


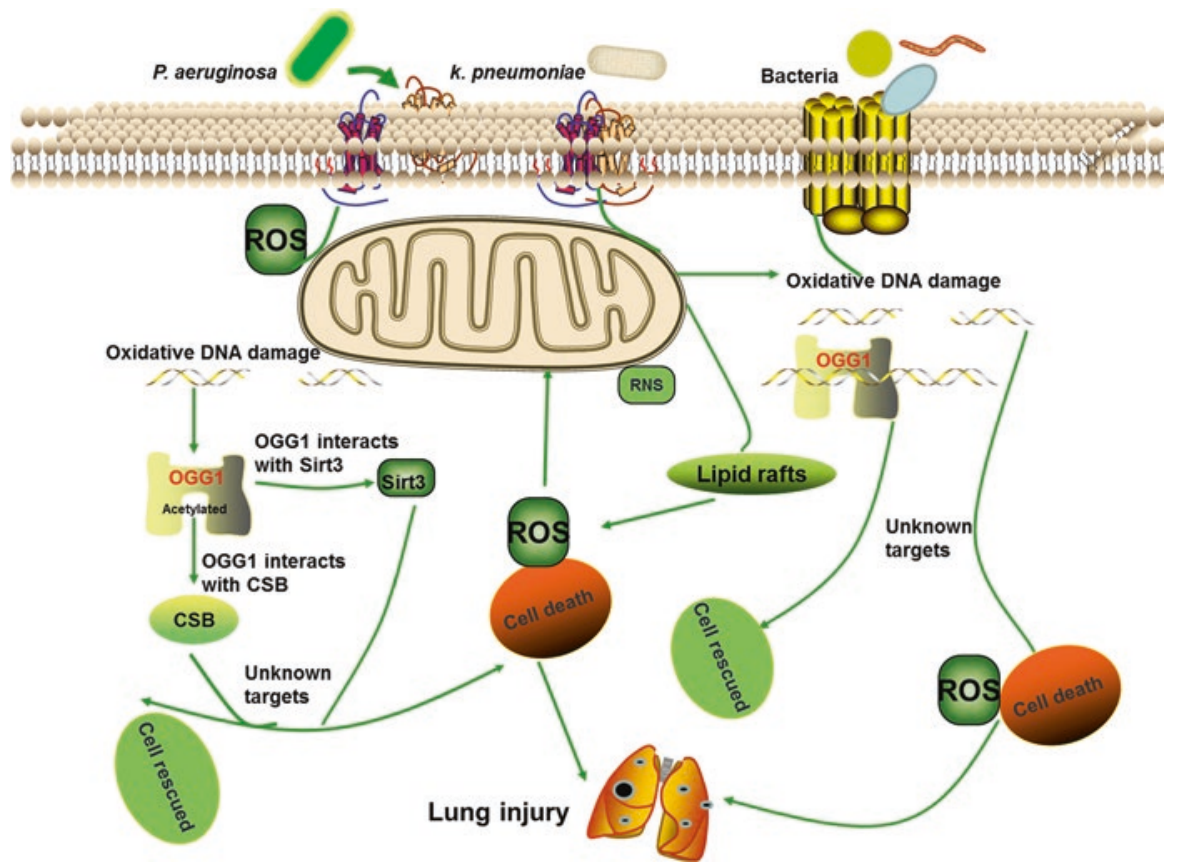

Fig. 4.1 OGG1 response $P$. aeruginosa and $K$. pneumoniae infection: $P$. aeruginosa infection induces ROS and subsequent oxidative DNA damage, which activates OGG1 acetylation. The acetylated OGG1 reacts with uncanonical CSB or Sirt3 pathways and repairs DNA damage to rescue host cells. In contrast, inhibition of OGG1 acetylation will result in cell death and lung injury. K. pneumoniae-induced oxidation elicits DNA damage following ROS production by activation of lipid rafts. This process can cause serious oxidative DNA damage that can be repaired by OGG1. The same mechanism is also implicated in other bacteria

\subsubsection{OGG1 Affects Viral Infection}

DNA damage-induced signaling has been recognized as a key factor in viral infection. Bovine herpesvirus-1 (BoHV-1), a bovine-derived virus that causes severe respiratory diseases and inflammation, can induce oxidative DNA damage and exhaust the expression level of OGG1 by activating the production of ROS. OGG1 can ameliorate DNA damage and inhibit viral replication to absorb the excessive ROS (Zhu et al. 2018). The titer of the hepatitis C virus (HCV) is inversely related to the expression of OGG1, as high viral burdens aggravate cellular DNA damage levels but suppressed damage-related DNA repair gene expression. qPCR and Western blotting showed that OGG1 levels were significantly upregulated in low viral level (LVL) cells but downregulated in high viral level (HVL) cells (Piciocchi et al. 2016). In patients with viral hepatitis, OGG1 polymorphism is associated with oxidative DNA damage and disease progression and patient survival rate, suggesting that OGG1 plays an important role in the pathophysiology of HCV and hepatitis B virus (HBV) infection (Jung et al. 2012). The proportion of HIV genes integrated 
into host cells is significantly reduced in murine cells with deletions of the gene Ogg1, indicating that the activity of the repair gene enzyme is mediated by BER pathway of oxidative DNA damage, which is essential for HIV viral infection (Yoder et al. 2011). Ground-glass hepatocytes (GGHs) are a sign of the late stage of HBV infection, expressing the pre-S 1 and pre-S 2, two mutant types of large HBV surface antigens (HBsAg). The HBV carrying pre-S mutation aggravates the infectious process by induction of cellular oxidative stress and DNA damage and the expression of OGG1 in hepatocellular carcinoma. These results indicate that ROSmediated DNA damage and OGG1-mediated repair are involved in the infection of HBV. Using SF91 lentiviral delivery, the overexpression of OGG1 ameliorates hematopoietic cell damage situation by repairing TEPA-induced DNA damage (Hsieh et al. 2004).

\subsubsection{OGG1 Affects Fungus Infection}

Fungal infections can also cause DNA damage similar to bacterial and viral infections. Proanthocyanidins can extenuate aflatoxin B1-induced oxidative DNA damage and carcinogenesis by regulation of the expression of $\mathrm{Oggl}$ (Bakheet et al. 2016). OGG1 has a different role in maintaining the genetic stability of endogenous genes and coping with exogenous stress. OGG1 can protect genetic stability by reducing the accumulation of endogenous mutagenic lesions. However, OGG1 can incite toxicity and mutation when 4-nitroquinoline-1-oxide (4-NQO) treatment induces a potent intracellular oxidative stress in a ccc2-deficient strain of $S$. cerevisiae (da Silva et al. 2015). Aflatoxin B1, a carcinogenic molecule in the process of Aspergillus fungal infection, can accelerate cancer development by increasing base excision repair activity and OGG1 levels in mouse lungs (Guindon-Kezis et al. 2014).

\subsection{OGG1 Is Linked to Inflammatory Lung Diseases}

\subsubsection{OGG1 Responses in Pulmonary Hyperoxia}

Standard oxygen therapy functions as a supportive strategy for curing acute respiratory distress syndrome (ARDS) patients, which provides oxygen into the lungs to maintain the normal function while exchanging carbon dioxide in the blood at pulmonary alveoli (Ragaller and Richter 2010). Although there is no direct cure for ARDS, supportive therapies by providing sufficient oxygen for the patient are critical measure to heal the lungs from the injury (Bulger et al. 2000). However, it should be cautioned that hyperoxia is also toxic to the lung that receives oxygen therapy (Kallet and Matthay 2013), which is a proverbial cause antecedent of injuring alveolar epithelial cells (AECs) to developing lung disease (Li et al. 2018). The activation of $\mathrm{p} 21$ - and $\mathrm{p} 53$-mediated signaling pathway produces similar results to those brought out by hyperoxia, resulting in extensive injury to AECs, especially 
type II AECs, and an elevated risk of jeopardizing the lungs' functional integrity (O'Reilly et al. 1998; McGrath-Morrow et al. 2011; Yee et al. 2011). The injury or death of type II AECs is a devastating event for the alveoli because the type II cell is considered as the progenitor of alveolar epithelial cells, which may disrupt surface tension at the air/liquid interface and prevent the collapse of alveoli (Whitsett and Alenghat 2015). DNA damage derived from high concentrations of oxygen is one of the hyperoxia-derived pathogenic factors that is responsible for AEC injury in lung tissues by creating vast amounts of ROS, resulting in excessive inflammation (Ye et al. 2017). Fortunately, the damage done to DNA during hyperoxia can be repaired by OGG1, preventing programmed death of lung epithelial cells ( $\emptyset_{\text {vrevik }}$ et al. 2010). Overexpression of OGG1 alleviates the toxic effect of hyperoxiaderived injury in lung cells (Wu et al. 2002). Therefore, enhancing expression levels of OGG1 functions represents a potential approach to defend the lung against hyperoxia-induced lung injury.

OGG1 plays an important role in hyperoxia-induced inflammatory response. A recent study showed that high concentrations of oxygen could result in excessive proinflammatory cytokine, such as TNF- $\alpha$, IL-6, and IFN- $\beta$, in Ogg 1 -deficient mice (Ye et al. 2017). Although not immediately evident, autophagy also plays a role in the regulation of inflammation associated with OGG1, along with a broad spectrum of DNA repair proteins (Ye et al. 2017; Van Houten et al. 2016). In cardiomyopathy, autophagy plays an important role in keeping a balance between ROS production and loss of OGG1(Ye et al. 2017; Ryter and Choi 2013). Moreover, pulmonary hyperoxia also stimulates autophagy, but OGG1-mediated control of autophagy results in reduced damage of lung tissues and inflammatory responses. The OGG1/ Atg7 axis inhibits NF- $\kappa \mathrm{B}$ phosphorylation process that exhibited attenuated inflammatory cytokines such as TNF- $\alpha$, IL- 6 , and IFN- $\beta$, demonstrating that OGG1 associates with autophagy to regulate inflammatory responses to pulmonary hyperoxia. Taken together, in pulmonary hyperoxia, OGG1 is associated with autophagic pathway to repress the production of inflammatory cytokines, which alleviates hyperoxiainduced lung tissue injury (Ye et al. 2017). These findings imply that OGG1 plays a significant role in innate immunity against hyperoxia as well as novel targets for clinical therapy.

\subsubsection{OGG1 Mediates Pulmonary Fibrosis}

Pulmonary fibrosis is a chronic and progressive respiratory disease, in which a scar is formed in distal lung interstitial tissue over time (Richeldi et al. 2017). The scar tissue is formed by excessive extracellular matrix collagen deposition following the injury of overlying epithelium and activation of local myofibroblasts (Darby et al. 2014). The scarring and consequent stiffening of the lungs make breathing difficult and cannot afford for sufficient oxygen supply to the blood. Asbestosis is one of the widespread pulmonary fibroses that is caused by asbestos exposure (Walters et al. 2018). Thus far, asbestos-related pulmonary fibrosis remains a common public health issue around the world. The injured AECs are associated with pulmonary 
fibrosis induced by asbestosis (Cheresh et al. 2015). Asbestos-associated AEC injury and inflammatory cells lead to ROS production to promote disease occurrence (Cheresh et al. 2015). In this process, mtDNA is more sensitive to crocidolite asbestos-induced DNA damage leading to the production of iron-derived free mitochondrial ROS and apoptosis (Kim et al. 2015). Moreover, the AEC-mediated apoptosis plays an important role in pulmonary fibrosis (Kim et al. 2015; Malsin and Kamp 2018). In addition, oxidative stress caused by asbestos exposure induces mitochondrial dysfunction by affecting mitochondrial ROS-mediated mtDNA damage, p53 activation, apoptosis, and inflammatory signaling (Shetty et al. 2017). It demonstrates that oxidative stress in asbestos fibrosis plays an important role for AEC-mediated mtDNA damage to promote the development of pulmonary fibrosis.

OGG1 is primarily responsible for mtDNA damage repair that facilitates ensuring of long-term cell survival, indicating that OGG1 as a bifunctional base excision repair protein shows importance for asbestos-induced pulmonary fibrosis (Cheresh et al. 2015). OGG1 mutant shows exacerbated lung fibrosis scores compared to WT mice. Moreover, ineffective base excision repair pathway results in increased mtDNA, and asbestos-induced AEC injury is strongly associated with apoptosis via p53 activation (Kim et al. 2015; Panduri et al. 2009, 2003, 2006). Furthermore, OGG1 deficiency augments caspase- 3 activation in lung AEC cells to aggravate the pulmonary fibrosis (Cheresh et al. 2015). In addition, asbestos also induces an increased endoplasmic reticulum (ER) stress response in $O g g 1^{-1-}$ mice. Taken together, OGG1-mediated prevention of asbestos-induced AEC mtDNA damage and apoptosis is through p53 pathway to protect cell integrity and alleviate the extent of pulmonary fibrosis (Liu et al. 2013). This demonstrates that lung AEC integrity maintained by OGG1 plays an important role in pulmonary fibrosis disease, which represents a novel and useful therapeutic approach.

\subsubsection{OGG1 Controls Allergic Airway Inflammatory Diseases}

Asthma is a complex, chronic inflammatory lung disease and is thought to be heavily influenced by genetics as well as epigenetic changes induced by environmental factors (Ho 2010; Lambrecht and Hammad 2012). The genetics, development, immunopathogenesis, and ventilator/gas exchange impairment associated with the disease are dictated by multiple inflammatory mediators in addition to the oxidative stress induced by ROS (Zuo et al. 2013).

Environmental agents, acting individually or in combination, mainly affect airway epithelium and immune cells through increasing the generation of ROS levels, leading to the beginning and worsening of asthma (Birben et al. 2012). ROS generation significantly enhances genomic 8-oxo-dG levels that are connected with OGG1 protein. Moreover, OGG1-initiated repair of damaged DNA is an indispensable for neutrophil accumulation that is implicated in deregulation of the immune system and fundamental to histopathological changes in allergic airway inflammatory disease (Bacsi et al. 2013; Ba et al. 2015). OGG1-dependent KRAS activation 
participates in the recruitment of airway allergic inflammatory cells and releases chemokine/cytokine Cxcl1, TNF- $\alpha, \mathrm{Ccl} 20, \mathrm{Ccl} 3, \mathrm{IL}-1 \alpha$, and IL-1 $\beta$ (AguileraAguirre et al. 2014). In addition, OGG1-driven MAPK, PI3K, and MSK1 pathways on NF- $\mathrm{kB} /$ RelA activation could also be linked to airway allergic inflammatory disease. It activates I $\mathrm{KB}$ phosphorylation that becomes a target for ubiquitination and proteasome-mediated degradation leading to liberation of NF-кB/RelA activation. Moreover, OGG1 deficiency also modifies allergic airway inflammation by alleviating the expression and phosphorylation of STAT6 and IL-4, IL-5, IL-6, IL-10, IL-13, and IL-7 but increasing IFN- $\gamma$ production in the lung tissue (Li et al. 2012). Taken together, OGG1 plays an important role in the regulation of allergic immune response via generating different endogenous signals for inflammation.

\subsubsection{OGG1 Response Is Related to Other Diseases}

OGG1 is etiologically linked with several other inflammatory diseases, including Alzheimer's disease, atherosclerosis, metabolic syndrome, rheumatoid arthritis, cancer, or diabetes. In the atherosclerosis disease, OGG1-mediated BER represses NLRP3 (NLR family pyrin domain containing 3) inflammasome activation and decreased apoptosis resulting in less IL- $1 \beta$ and IL-18 secretion to prevent the disease progression of atherosclerosis (Tumurkhuu et al. 2016; Bennett et al. 2018). It shows that OGG1 functions as a protective effector on atherosclerosis by restricting excessive inflammasome activation (Baldrighi et al. 2017). Alteration of OGG1BER is an event preceding Alzheimer's disease so that the level of OGG1 as a potential diagnostic biomarker contributes to neuronal cell death in dementia (Sanyal 2007). On the other hand, single nucleotide polymorphism (SNP) of Oggl gene associated with the control of the level of oxidative DNA damage has a notable role on the development of rheumatoid arthritis (Yosunkaya et al. 2012), cancer (Moghaddam et al. 2016), and Alzheimer's disease (Kwiatkowski et al. 2016). For example, increased rheumatoid arthritis risk is linked to $\mathrm{Oggl} \mathrm{C} 285 \mathrm{~T}$ and $\mathrm{A} 5954 \mathrm{G}$ polymorphisms. Moreover, OGG1 is associated with low rates of obesity and Syndrome X (metabolic syndrome), due to its role in monitoring energy and fat balance, especially the alteration of lipid and mitochondrial metabolism (Vartanian et al. 2017). Lastly, in the type II diabetes disease, OGG1 is involved in AMPKmediated NRF2 signals, which diminishes renal cell destruction by minimizing oxidative DNA damage (Habib et al. 2016). Overall, it is clear that the novel discovery of OGG1-mediated DNA repair has broad implications for the determination of multiple human disease mechanisms.

\subsection{Conclusion and Perspective}

Genomic instability is a hallmark of disease that increases due to the alteration of DNA damage response. OGG1 localizes in both nucleus and mitochondria and has been investigated for its role in DNA repair in many pathways modulating 
pulmonary infection and other inflammatory responses. We highlighted OGG1mediated DNA repair mechanisms that impact ROS production, DNA damage, p21/ p53 activation, apoptosis, and autophagy. These researches provide insights into the molecular basis of OGG1-associated signaling in bacterial infection and inflammation, which may foster the development of novel therapeutic strategies for infection, degenerative disease, tumors, and aging. The OGG1/pulmonary disease paradigm will shed light into the molecular basis underlying diseases in other organs, such as the liver, heart, and kidney, which will provide an improved understanding of the pathogenesis of many common diseases, for which effective treatment regiments are urgently required. Furthermore, we may witness a flux of novel discoveries in elaborating basic principles of how OGG1 regulates inflammatory response and other cell signalings, such as the molecular binding partners, transcriptional control, promoter activation, and structural alterations in various disease conditions.

Funding This work was supported by the National Institutes of Health 5R01AI109317-04, 1R01AI138203-01, P20GM103442, and P20GM113123. This work was also supported by the Key Program of the National Nature Science Foundation of China (81530063) to Jianxin Jiang. The funders had no role in study design, data collection and analysis, decision to publish, or preparation of the manuscript.

\section{References}

Adriani A, Repici A, Hickman I, Pellicano R (2014) Helicobacter pylori infection and respiratory diseases: actual data and directions for future studies. Minerva Med 105:1-8

Aguilera-Aguirre L et al (2014) Innate inflammation induced by the 8-oxoguanine DNA glycosylase-1-KRAS-NF-kB pathway. J Immunol:1401625

Ba X et al (2014) The role of 8-oxoguanine DNA glycosylase-1 in inflammation. Int J Mol Sci 15:16975-16997

Ba X, Aguirre LA, Sur S, Boldogh I (2015) 8-oxoguanine DNA glycosylase-1 driven DNA base excision repair: role in asthma pathogenesis. Curr Opin Allergy Clin Immunol 15:89

Bacsi A et al (2013) Down-regulation of 8-oxoguanine DNA glycosylase 1 expression in the airway epithelium ameliorates allergic lung inflammation. DNA Repair 12:18-26

Bakheet SA et al (2016) Alleviation of Aflatoxin B1-Induced Genomic Damage by Proanthocyanidins via Modulation of DNA Repair. J Biochem Mol Toxicol 30:559-566

Baldrighi M, Mallat Z, Li X (2017) NLRP3 inflammasome pathways in atherosclerosis. Atherosclerosis 267:127

Bartz RR et al (2011) Staphylococcus aureus sepsis and mitochondrial accrual of the 8-oxoguanine DNA glycosylase DNA repair enzyme in mice. Am J Respir Crit Care Med 183:226-233. https://doi.org/10.1164/rccm.200911-1709OC

Bartz RR et al (2014) Staphylococcus aureus sepsis induces early renal mitochondrial DNA repair and mitochondrial biogenesis in mice. PLoS One 9:e100912. https://doi.org/10.1371/journal. pone. 0100912

Bennett MR, Shah AV, Gray K, Starks L (2018) Defective Base Excision Repair of Oxidative DNA Damage in Vascular Smooth Muscle Cells Promotes Atherosclerosis. Atheroscler Suppl 32:5

Birben E, Sahiner UM, Sackesen C, Erzurum S, Kalayci O (2012) Oxidative stress and antioxidant defense. World Allergy Organ J 5:9

Bulger EM, Jurkovich GJ, Gentilello LM, Maier RV (2000) Current clinical options for the treatment and management of acute respiratory distress syndrome. J Trauma 48:562-572 
Cheng Y et al (2013) Interaction of Sirt3 with OGG1 contributes to repair of mitochondrial DNA and protects from apoptotic cell death under oxidative stress. Cell Death Dis 4:e731. https:// doi.org/10.1038/cddis.2013.254

Cheresh P et al (2015) Asbestos-induced pulmonary fibrosis is augmented in 8-oxoguanine DNA glycosylase knockout mice. Am J Respir Cell Mol Biol 52:25-36

da Silva CR, Almeida GS, Caldeira-de-Araújo A, Leitão AC, de Pádula M (2015) Influence of Ogg1 repair on the genetic stability of ccc2 mutant of Saccharomyces cerevisiae chemically challenged with 4-nitroquinoline-1-oxide (4-NQO). Mutagenesis 31:107-114

Darby IA, Laverdet B, Bonte F, Desmouliere A (2014) Fibroblasts and myofibroblasts in wound healing. Clin Cosmet Investig Dermatol 7:301-311. https://doi.org/10.2147/CCID.S50046

Deng B, Li Y, Zhang Y, Bai L, Yang P (2013) Helicobacter pylori infection and lung cancer: a review of an emerging hypothesis. Carcinogenesis 34:1189-1195. https://doi.org/10.1093/ carcin/bgt114

Ewald CY (2018) Redox signaling of NADPH oxidases regulates oxidative stress responses, immunity and aging. Antioxidants (Basel) 7. https://doi.org/10.3390/antiox7100130

Guindon-Kezis KA, Mulder JE, Massey TE (2014) In vivo treatment with aflatoxin B1 increases DNA oxidation, base excision repair activity and 8-oxoguanine DNA glycosylase 1 levels in mouse lung. Toxicology 321:21-26

Habib SL, Yadav A, Kidane D, Weiss RH, Liang S (2016) Novel protective mechanism of reducing renal cell damage in diabetes: activation AMPK by AICAR increased NRF2/OGG1 proteins and reduced oxidative DNA damage. Cell Cycle 15:3048-3059

Ho S-M (2010) Environmental epigenetics of asthma: an update. J Allergy Clin Immunol 126:453-465

Hsieh Y-H et al (2004) Pre-S mutant surface antigens in chronic hepatitis B virus infection induce oxidative stress and DNA damage. Carcinogenesis 25:2023-2032

Huang $\mathrm{H}$ et al (2013) Lipid-based signaling modulates DNA repair response and survival against Klebsiella pneumoniae infection in host cells and in mice. Am J Respir Cell Mol Biol 49:798807. https://doi.org/10.1165/rcmb.2013-0069OC

Izzotti A et al (2007) Interplay between Helicobacter pylori and host gene polymorphisms in inducing oxidative DNA damage in the gastric mucosa. Carcinogenesis 28:892-898. https:// doi.org/10.1093/carcin/bgl208

Jung SW et al (2012) Polymorphisms of DNA repair genes in Korean hepatocellular carcinoma patients with chronic hepatitis B: possible implications on survival. J Hepatol 57:621-627

Kallet RH, Matthay MA (2013) Hyperoxic acute lung injury. Respir Care 58:123-141. https://doi. org/10.4187/respcare.01963

Kate V, Ananthakrishnan N, Tovey FI (2013) Is Helicobacter pylori Infection the Primary cause of duodenal ulceration or a secondary factor? A review of the evidence. Gastroenterol Res Pract 2013:425840. https://doi.org/10.1155/2013/425840

Kim S-J, Cheresh P, Jablonski R, Williams D, Kamp D (2015) The role of mitochondrial DNA in mediating alveolar epithelial cell apoptosis and pulmonary fibrosis. Int J Mol Sci 16:21486-21519

Kuck JL et al (2015) Mitochondrial DNA damage-associated molecular patterns mediate a feedforward cycle of bacteria-induced vascular injury in perfused rat lungs. Am J Physiol Lung Cell Mol Physiol 308:L1078-L1085. https://doi.org/10.1152/ajplung.00015.2015

Kwiatkowski D et al (2016) Association between single-nucleotide polymorphisms of the hOGG1, NEIL1, APEX1, FEN1, LIG1, and LIG3 genes and Alzheimer's disease risk. Neuropsychobiology 73:98-107

Lambrecht BN, Hammad H (2012) The airway epithelium in asthma. Nat Med 18:684

Lee YL et al (2017) Mitochondrial DNA damage initiates acute lung injury and multi-organ system failure evoked in rats by intra-tracheal Pseudomonas Aeruginosa. Shock 48:54-60. https://doi.org/10.1097/SHK.0000000000000838

Li G et al (2012) 8-Oxoguanine-DNA glycosylase 1 deficiency modifies allergic airway inflammation by regulating STAT6 and IL-4 in cells and in mice. Free Radic Biol Med 52:392-401 
Li PS, Tao W, Yang LQ, Shu YS (2018) Effect of soluble epoxide hydrolase in hyperoxic acute lung injury in mice. Inflammation 41:1065-1072. https://doi.org/10.1007/s10753-018-0758-y

Liu G, Cheresh P, Kamp DW (2013) Molecular basis of asbestos-induced lung disease. Annu Rev Pathol: Mech Dis 8:161-187

Liu S et al (2018) IRF-1 Intervention in the Classical ROS-Dependent Release of NETs during LPS-Induced Acute Lung Injury in Mice. Inflammation:1-17

Malsin ES, Kamp DW (2018) The mitochondria in lung fibrosis: friend or foe? Transl Res 202:1

Mathieu A, O'Rourke EJ, Radicella JP (2006) Helicobacter pylori genes involved in avoidance of mutations induced by 8-oxoguanine. J Bacteriol 188:7464-7469. https://doi.org/10.1128/ JB.00851-06

McGrath-Morrow SA et al (2011) Neonatal hyperoxia contributes additively to cigarette smokeinduced chronic obstructive pulmonary disease changes in adult mice. Am J Respir Cell Mol Biol 45:610-616. https://doi.org/10.1165/rcmb.2010-0259OC

Moghaddam AS, Nazarzadeh M, Noroozi R, Darvish H, Jarrahi AM (2016) XRCC1 and OGG1 gene polymorphisms and breast cancer: a systematic review of literature. Iranian J Cancer Prev 9

O'Reilly MA, Staversky RJ, Stripp BR, Finkelstein JN (1998) Exposure to hyperoxia induces p53 expression in mouse lung epithelium. Am J Respir Cell Mol Biol 18:43-50. https://doi. org/10.1165/ajrcmb.18.1.2950m

Øvrevik J et al (2010) Differential effects of nitro-PAHs and amino-PAHs on cytokine and chemokine responses in human bronchial epithelial BEAS-2B cells. Toxicol Appl Pharmacol 242:270-280

Panduri V, Weitzman SA, Chandel N, Kamp DW (2003) The mitochondria-regulated death pathway mediates asbestos-induced alveolar epithelial cell apoptosis. Am J Respir Cell Mol Biol 28:241-248

Panduri V et al (2006) P53 Mediates amosite asbestos-induced alveolar epithelial cell mitochondria-regulated apoptosis. Am J Respir Cell Mol Biol 34:443-452

Panduri V et al (2009) Role of mitochondrial hOGG1 and aconitase in oxidant-induced lung epithelial cell apoptosis. Free Radic Biol Med 47:750-759

Piciocchi M et al (2016) Differential timing of oxidative DNA damage and telomere shortening in hepatitis C and B virus-related liver carcinogenesis. Transl Res 168:122-133

Ragaller M, Richter T (2010) Acute lung injury and acute respiratory distress syndrome. J Emerg Trauma Shock 3:43-51. https://doi.org/10.4103/0974-2700.58663

Richeldi L, Collard HR, Jones MG (2017) Idiopathic pulmonary fibrosis. Lancet 389:1941-1952

Ryter SW, Choi AM (2013) Regulation of autophagy in oxygen-dependent cellular stress. Curr Pharm Des 19:2747-2756

Sanyal S (2007) Effect of genetic polymorphisms on urinary bladder neoplasms. (Institutionen för onkologi-patologi/Department of Oncology-Pathology)

Shetty SK et al (2017) p53 and miR-34a Feedback promotes lung epithelial injury and pulmonary fibrosis. Am J Pathol 187:1016-1034. https://doi.org/10.1016/j.ajpath.2016.12.020

Stehle C, Hernández DC, Romagnani C (2018) Innate lymphoid cells in lung infection and immunity. Immunol Rev 286:102-119

Testa G, Rossin D, Poli G, Biasi F, Leonarduzzi G (2018) Implication of oxysterols in chronic inflammatory human diseases. Biochimie 153:220

Touati E et al (2006) Deficiency in OGG1 protects against inflammation and mutagenic effects associated with $\mathrm{H}$. pylori infection in mouse. Helicobacter 11:494-505. https://doi. org/10.1111/j.1523-5378.2006.00442.x

Tumurkhuu G et al. (2016) Ogg1-dependent DNA repair regulates NLRP3 inflammasome and prevents atherosclerosis. Circ Res, CIRCRESAHA 116.308362

Van Houten B, Hunter SE, Meyer JN (2016) Mitochondrial DNA damage induced autophagy, cell death, and disease. Front Biosci (Landmark edition) 21:42

Vartanian V et al (2017) 8-oxoguanine DNA glycosylase (OGG1) deficiency elicits coordinated changes in lipid and mitochondrial metabolism in muscle. PLoS One 12:e181687 
Vlahopoulos S, Adamaki M, Khoury N, Zoumpourlis V, Boldogh I (2018) Roles of DNA repair enzyme OGG1 in innate immunity and its significance for lung cancer. Pharmacol Ther

Walters G, Robertson A, Bhomra P, Burge P (2018) Asbestosis is prevalent in a variety of construction industry trades. NPJ Prim Care Respir Med 28:11

Wang R et al (2018) OGG1-initiated base excision repair exacerbates oxidative stress-induced parthanatos. Cell Death Dis 9(628)

Whitsett JA, Alenghat T (2015) Respiratory epithelial cells orchestrate pulmonary innate immunity. Nat Immunol 16:27-35. https://doi.org/10.1038/ni.3045

Wu M et al (2002) Protection of human lung cells against hyperoxia using the DNA base excision repair genes hOgg1 and Fpg. Am J Respir Crit Care Med 166:192-199

Wu M et al (2011) Host DNA repair proteins in response to Pseudomonas aeruginosa in lung epithelial cells and in mice. Infect Immun 79:75-87

Ye Y et al (2017) DNA repair interacts with autophagy to regulate inflammatory responses to pulmonary hyperoxia. J Immunol 198:2844-2853. https://doi.org/10.4049/jimmunol.1601001

Yee M et al (2011) Neonatal hyperoxia causes pulmonary vascular disease and shortens life span in aging mice. Am J Pathol 178:2601-2610. https://doi.org/10.1016/j.ajpath.2011.02.010

Yoder KE et al (2011) The base excision repair pathway is required for efficient lentivirus integration. PLoS One 6:e17862

Yosunkaya E et al (2012) Rheumatoid arthritis risk associates with DNA repair gene XRCC1 Arg399Gln polymorphism in Turkish patients. Rheumatol Int 32:1265-1269

Yuan D et al (2017) Kupffer cell-derived Tnf triggers cholangiocellular tumorigenesis through JNK due to chronic mitochondrial dysfunction and ROS. Cancer Cell 31:771-789.. e776

Zhang S et al (2017) Nitric oxide synthase activity correlates with OGG1 in ozone-induced lung injury animal models. Front Physiol 8(249)

Zhang H et al (2018) Reactive oxygen species stimulated pulmonary epithelial cells mediate the alveolar recruitment of FasL+ killer B cells in LPS-induced acute lung injuries. J Leukoc Biol 104:1187

Zhu L, Fu X, Yuan C, Jiang X, Zhang G (2018) Induction of Oxidative DNA Damage in Bovine Herpesvirus 1 Infected Bovine Kidney Cells (MDBK Cells) and Human Tumor Cells (A549 Cells and U2OS Cells). Viruses 10:393

Zuo L, Otenbaker NP, Rose BA, Salisbury KS (2013) Molecular mechanisms of reactive oxygen species-related pulmonary inflammation and asthma. Mol Immunol 56:57-63 